\title{
Development Of An Atherothrombotic Occlusion In The Rabbit Carotid Artery: Accessed By New Computerized B- Mode Ultrasound Image Processing Technology And Histopathology
}

\author{
Hossein Mehrad ${ }^{1}$, M ehdi Farhoudi ${ }^{2}$, Ehsan Sharifipour ${ }^{3}$, Behyad Mahvi ${ }^{4}$ \\ ${ }^{1}$ Islamic Azad University, Tabriz Branch, Faculty of Basic Sciences, Department of Physics, Tabriz, Iran. \\ ${ }^{2}$ Tabriz University of M edical Sciences, Neurosceinces Research Center (NSRC). \\ ${ }^{3}$ Qom University of M edical Sciences, School of M edicine, Department of Neurology, Qom, Iran. \\ ${ }^{4}$ Islamic Azad University, Tabriz Branch, Faculty of M echanic, Department of Bioengineering, Tabriz, Iran.
}

Introduction: Thrombus formation on a disrupted atherosclerotic soft plaque is a key event that leads to atherothrombosis. Atherothrombosis is one of the leading causes of acute coronary syndrome and ischemic stroke. Our ability to test new protocols for the treatment of atherothrombotic stenosis in humans is limited for obvious ethical reasons; therefore, a precise understanding of the mechanism of atherothrombotic occlusion in human carotid artery, which give rise to thrombosis, emboli and stroke, requires a suitable animal model that would mimic the same characteristics well.

Aims: The aim of this study was to generate an easily reproducible and inexpensive experimental rabbit carotid model of atherothrombotic occlusion with morphological similarities to the human disease and the subsequent assessment of the reliability of new computerized B- mode ultrasound image processing technology in the study of lumen area stenosis in this model.

Methods: Briefly, male New Zealand white rabbits were submitted to common carotid artery atherothrombotic occlusion by primary balloon injury followed $1.5 \%$ cholesterol- rich diet injury for eight weeks and finally perivascularly severe cold injury. All of the rabbits' arteries were imaged by B-mode ultrasound weekly, after which the rabbits were sacrificed, and their vessels were processed for histopathology. Ultrasound longitudinal view images from three cardiac cycles were processed by a new computerized analyzing method based on dynamic programming and maximum gradient algorithm for measurement of instantaneous changes in arterial wall thickness and lumen diameter in sequential ultrasound images.

Results: Histopathology results showed progressive changes, from the lipid-laden cells and fibrous connective tissue proliferation, fibrolipid plaque formation, resulting in vessel wall thickening, remodeling, neovascularization and lumen narrowing (before perivascularly severe cold injury using liquid nitrogen) up to atherothrombotic occlusion (after perivascularly severe cold injury using liquid nitrogen) in the treatment group respect to other groups $(P<0.05)$.

Conclusion: In conclusion we successfully developed a new rabbit carotid artery model of atherothrombotic occlusion, which is not only quickly and easily reproducible and inexpensive, but also without mortality and similar to the condition seen in patients. The merits of our model render the evaluation of carotid artery atherothrombotic stenosis and its treatment strategies more feasible in humans. This condition in rabbits can be properly assessed by our new computerized B-mode ultrasound image processing technology.

Key words: atherothrombotic, rabbit carotid, ultrasound

DOI: 10.7575/aiac.abcmed.ca1.59

A I Published Date: February 2017

Peer-review is under responsibility of the 9th Iranian Stroke Congress.

Published by Australian International Academic Centre, Australia

This published work is open access under the CC BY license.

Available online at www.abcmed.aiac.org.au 